\title{
A Hybrid-CPU-FPGA-based Solution to the Recovery of Sha256crypt-hashed Passwords
}

\author{
Zhendong Zhang and Peng Liu* \\ College of Information Science and Electronic Engineering, \\ Zhejiang University, Hangzhou, China \\ \{zhendong404, liupeng\}@zju.edu.cn
}

\begin{abstract}
This paper presents an accelerator design for the password recovery of sha256crypt based on hybrid CPU-FPGA devices. By applying the brute-force attack computation model proposed in this paper, we decompose the sha256crypt function into two types of operations, namely the data dispatching and the block transforming. The data dispatching operation generates message blocks and the block transforming operation transforms message blocks into digests. These two operations are efficiently accelerated by the customized data dispatch unit and the pipelined block transform unit, respectively. Difficulties of adopting the pipeline technique are addressed also with the following techniques. The group scheduling is used to solve the data dependency that stalls the pipeline. The look-ahead execution eliminates the uncertainty of the execution path. The data path pruning and spatial-temporal multiplexing reduce the resource overhead of non-computing units.

The proposed accelerator design is implemented and evaluated on the Xilinx Zynq7000 XC7Z030-3 SoC. Our experimental results show that the proposed accelerator can improve energy efficiency by $2.54 \times$ over the state-of-the-art password recovery tool Hashcat running on an NVIDIA GTX1080Ti GPU. Compared with the pure FPGA-based implementation in John-the-Ripper, the proposed accelerator improves energy efficiency by $1.64 \times$ and improves resource efficiency by $1.69 \times$.
\end{abstract}

Keywords: Password recovery · Linux security · FPGA · sha256crypt · key derivation function

\section{Introduction}

Password-based user authentication [Lam81] is the most commonly used method to protect user's important information, since passwords are highly portable and easy to understand by users. To prevent the clear text password from being easily obtained by malicious attackers, most operating systems and applications apply key derivation functions (KDFs) to convert clear text passwords to password hashes. Since KDFs are one-way functions, the only way to recover the clear text password from the password hash is the brute-force attack, which searches the correct password by performing KDF on all possible passwords until the output of KDF matches the password hash [Mar08]. A typical diagram of the brute-force attack is shown in Figure 1. The time consumption of brute-force attack depends on the searching speed and the size of the searching space. As the searching space is huge in most scenarios, malicious attackers have to pay enormous time and energy cost, and it is the same for the users who want to retrieve forgotten passwords. To expedite password recovery with a reasonable amount of time and energy consumption, a fast and energy-efficient accelerator becomes necessary.

\footnotetext{
*Corresponding author: Peng Liu.
} 


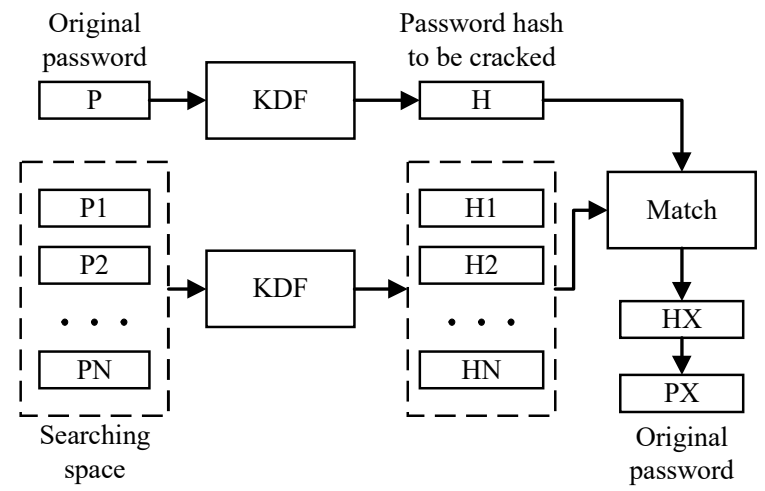

Figure 1: A typical diagram of the brute-force attack. The original password $P$ is encrypted by the KDF to generate a password hash $H$. The attacker applies KDF to all passwords in the searching space and matches every generated hash with the password hash $H$. If $H X$ matches the password hash $H$, the original password $P X$ is retrieved.

As the details of different KDFs diverse from each other, for the sake of illustration, common features of the brute-force attack on KDFs are abstracted and summarized in Figure 2. A KDF thread repeatedly generates a message block from the password or the previous digests, then transforms it to a new digest. Due to the data dependency between two adjacent message blocks, blocks in a single KDF thread must be processed sequentially. As a result, many researchers aim at increasing the parallelism of hardware accelerators, so that more passwords could be processed concurrently.

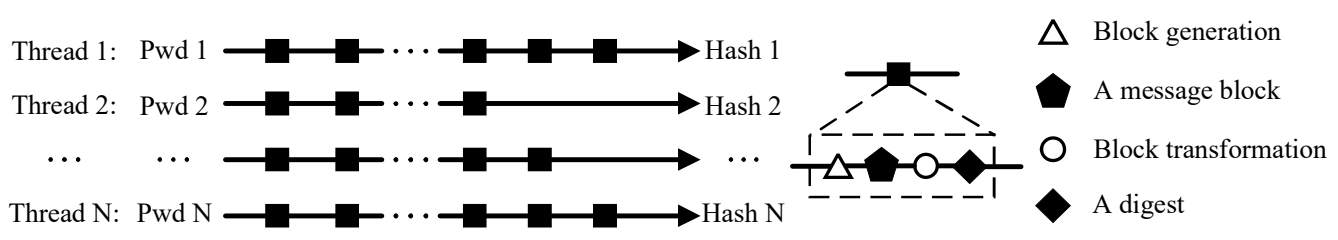

Figure 2: Computation model of the brute-force attack on KDFs. N passwords are processed concurrently with N KDF threads. Each block depends on its previous block. All block transform operations cost the same time. The number of blocks may vary for different passwords.

There are two types of parallelism in the brute-force attack of KDFs, the inter-block parallelism and the intra-block parallelism. Both of them are important to the design of accelerators. The inter-block parallelism refers to the number of blocks that the accelerator can process at the same time. The intra-block parallelism refers to the number of operations that the accelerator can perform at the same time during the processing of one block, which is the reciprocal of the number of cycles to process one block.

Among many of the password recovery platforms, graphic process units (GPUs) and field programmable gate arrays (FPGAs) are most widely used. GPU-based accelerators $\left[\mathrm{QGG}^{+}\right.$16, HK17, AKSE18] utilize thousands of processing cores to achieve a high interblock parallelism. However, suffering from its general-purpose architecture, GPU costs thousands of cycles to process one block with high clock frequency, which makes it inferior to FPGA-based accelerators in terms of energy efficiency.

FPGA-based accelerators $\left[\mathrm{KMB}^{+} 16, \mathrm{LCL}^{+} 16, \mathrm{AVWS} 14, \mathrm{DGK}^{+} 12\right]$, on the other hand, can provide significant savings in terms of energy consumption [DK14]. They can process 
one block in dozens of cycles with customized high intra-block parallelism hash units. The customized hash units can also explore the inter-block parallelism by implementing a deep pipeline. The problem is that most FPGA-based accelerators only focus on password-based key derivation functions (PBKDF, PBKDF2), which have unified execution paths and regular block generation patterns. For other KDFs such as sha256crypt, sha512crypt, and md5crypt, the nonunified execution paths and complex block generation patterns make the implementation of pipelined hash units very difficult. Although the accelerator in [Mag18] implements sha256crypt with non-pipelined multi-cycle hash units, it allocates too many hardware resources for the complex control logic, which restricts its inter-block parallelism due to the limited hardware resources available on an FPGA.

There are three major difficulties when applying the pipelined hash units on KDFs like sha256crypt.

1. Dependency between adjacent blocks may stall the pipeline.

2. The unpredictable number of blocks for different passwords leads to nonunified execution paths and adds difficulties to the scheduling of pipeline.

3. The block generation patterns are related to the lengths of passwords, which complicate the memory access patterns and the design of the data path.

The emergence of hybrid CPU-FPGA devices [Xil16, Int20] provides a balance solution to address above difficulties in terms of flexibility and energy efficiency. It shows great performance on energy efficiency when employed to the password recovery of bcrypt [MK14] and PBKDF2 [LLD19]. Specifically, for the password recovery of sha256crypt, the CPU could handle the tasks with complex logic such as password generation and calculations that have nonunified execution paths. Then the FPGA is dedicated to the computationally intensive tasks which have unified execution paths and can be accelerated by the pipelined hash units.

To apply the pipeline technique on KDFs like sha256crypt, we propose an accelerator design based on hybrid CPU-FPGA devices. We analyze the sha256crypt function with our brute-force attack computation model and identify two basic operations. One is the data dispatching and another is the block transforming. The data dispatching operation generates blocks and the block transforming operation transforms blocks into digests, which are used to generate the subsequent blocks. With the definition of these two operations, it's obvious that an accelerator design should make customized hardware to expedite them efficiently. Following this guideline, several accelerating cores are implemented on the FPGA and work together with the CPU. Each accelerating core has three key components: the data buffers, the data dispatch unit, and the SHA256 block transform unit with a deep pipeline. Exploiting the co-processing of CPU and the reconfigurability of FPGA, several techniques, such as group scheduling, look-ahead execution, data path pruning, and spatial-temporal multiplexing, are applied to improve the performance and reduce the hardware resource overhead.

1. Group scheduling is used to remove the data dependency between adjacent blocks in sha256crypt so that the pipelined block transform unit is free from stalling.

2. Look-ahead execution eliminates the uncertainty of execution paths in sha256crypt by mapping the nonunified calculations to the CPU and reusing its results.

3. Data path pruning and spatial-temporal multiplexing are used to simplify the design of the data dispatch unit and reduce the hardware resource overhead.

To the best of our knowledge, this is the first open work about the pipelined password recovery accelerator dedicated for the sha256crypt algorithm. The proposed accelerator is evaluated on a tailor-made development board with a Xilinx Zynq-7000 XC7Z030-3 SoC. 
Two accelerating cores are implemented based on the hardware resource available on the FPGA. The proposed accelerator achieves $2.54 \times$ improvement in terms of energy efficiency over the state-of-the-art implementation [has20] running on an NVIDIA GTX 1080Ti GPU. Compared with the pure FPGA-based accelerator reported in [Mag18], the proposed accelerator achieves $1.64 \times$ energy efficiency and $1.69 \times$ resource efficiency, respectively. The techniques proposed in this paper are also validated with sha512crypt. It achieves $2.39 \times$ energy efficiency over Hashcat running on GTX1080Ti.

The rest of this paper is structured as follows. Section 2 analyzes the details of sha256crypt and points out the difficulties in hardware implementation. Section 3 illustrates the overall architecture of the password recovery on a hybrid CPU-FPGA device. The details of the techniques employed are provided in Section 4. Section 5 gives the evaluation results of our design and Section 6 concludes the paper.

\section{Algorithm Details and Hardware Platforms}

\subsection{SHA256}

SHA256 function takes a message of 1-bit to $\left(2^{64}-1\right)$ bits as its input and outputs a digest of 256 bits. Inside the function, the input message is divided into several 512-bit blocks and processed block by block. Figure 3 illustrates the process of SHA256 function. The input message is first padded with a ' 1 ' followed by a stream of ' 0 's and a 64 -bit message length $L$ so that the total length is a multiple of 512 bits. Then starting from the first block, each block is processed together with a 256-bit state by the block transform function (BTF). Each block transform function outputs a 256-bit temporary digest, which is used as the state of the next block. The initialization vector (IV) is defined by the SHA-2 standard in [Dan15]. The final output of SHA256 is the digest of the last block $\left(\mathrm{D}_{\mathrm{N}}\right)$.

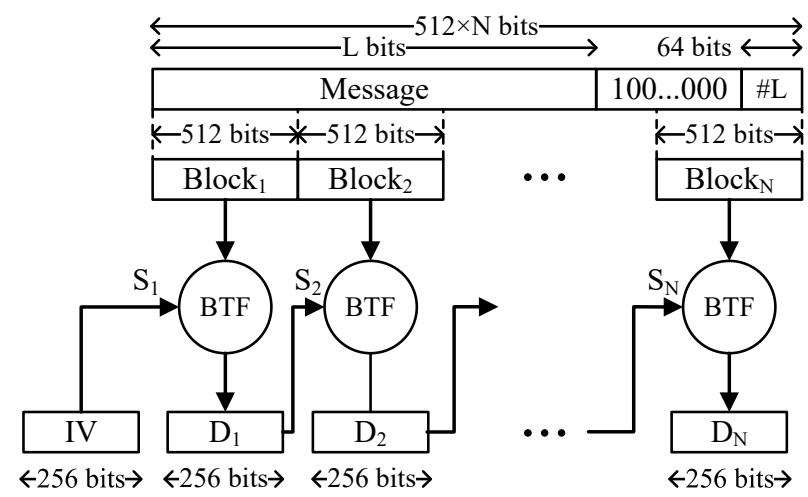

Figure 3: Details of the SHA256 function. BTF stands for the block transform function.

\subsection{Sha256crypt}

Sha256crypt is used in most Linux distributions to encrypt users' passwords. It is specified as follows:

$$
H=\operatorname{sha} 256 \operatorname{crypt}(p w d, \text { salt }, N)
$$

where $p w d$ is the password to be encrypted, salt is a random string of length 8 to 16 bytes, and $N$ is the number of SHA256 iterations in the function to adjust the execution time. The password hash $H$ contains four parts that are separated by the '\$' sign. An example of $H$ is shown below: 
$\$ 5 \$$ rounds $=5000 \$$ ssaalltt $\$$ Un $/ 5 j z A H M g O G Z 5 . m W J p u V o l i 107 g u H P v O W 8$

The ID ' 5 ' is reserved for sha256crypt. The number of iterations is specified in the second part with a default value of 5000. The third part is the salt string and the last part is the base64 [Jos06] encoded check value.

The process of sha256crypt can be divided into the following five phases, where the $L P$ and $L S$ denote the length of $p w d$ and salt, respectively.

Phase I:

1. Generate the message $A$ by consequently appending $p w d$, salt, and $p w d$. 2. Calculate the SHA256 digest of $A(D A)$.

Phase II:

1. Generate the message $B$ by consequently appending $p w d$, salt, and the first \#LP bytes of a sequence of $D A$ s. 2. For each digit $x$ of the binary representation of \#LP, starting from the lowest digit to the highest 1-digit:

(i) If $x$ is ' 1 ', append $D A$ to the message $B$.

(ii) If $x$ is ' 0 ', append $p w d$ to the message $B$.

3. Calculate the SHA256 digest of $B(D B)$.

Phase III:

1. Generate the message $P$ by consequently appending $p w d \# L P$ times. 2. Calculate the SHA256 digest of $P(D P)$. 3. Generate the message $S$ by consequently appending salt $16+D B[0]$ times, where the $D B[0]$ represents the first byte in $D B$ interpreted as an 8-bit unsigned value. 4. Calculate the SHA256 digest of $S(D S)$.

Phase IV:

1. Generate temporary variables $T P$ and $T S$ by intercepting the first \#LP bytes of a sequence of $D P \mathrm{~s}$ and the first \#LS bytes of a sequence of $D S \mathrm{~s}$, respectively. 2. Initialize the digest of $C(D C)$ with $D B$. 3. For $i$ ranging from 0 to $N$-1, repeat:

(1) Generate the message $C$ with the CryptPad function, which is detailed in Algorithm 1.

(2) Calculate the SHA256 digest of $C(D C)$.

\section{Phase V:}

1. Encode $D C$ with the base64 algorithm. 2. Generate the final output $H$.

As Phase V can be easily reversed, for the sake of representation, it will be omitted in this paper.

The security of sha256crypt mainly comes from three aspects. First, using random salt makes it impossible to apply rainbow table based attacks [Oec03]. Second, every SHA256 calculation in Phase IV-3 depends on the output of the previous SHA256 calculation. As a result, these calculations must be executed sequentially, which limits the processor's parallelism in a single thread of sha256crypt. Third, the iteration in Phase IV-3 with 1,000 to $999,999,999$ rounds increases computing power to defend against brute-force attacks.

\subsection{Sha256crypt in Brute-force Attack Computation Model}

As the number of blocks in SHA256 function is related to the length of the input message, different SHA256 calculations in sha256crypt may need different number of clock cycles to complete, which leads to the difficulties in the design of hardware architecture. To further explore the features of sha256crypt and make an efficient hardware implementation, the procedure of sha256crypt is decomposed to the block level by combining those described in Section 2.1 and Section 2.2.

As shown in Algorithm 1, every SHA256 calculation in sha256crypt is expressed as a loop of generating and transforming blocks. The generation and padding of the message 


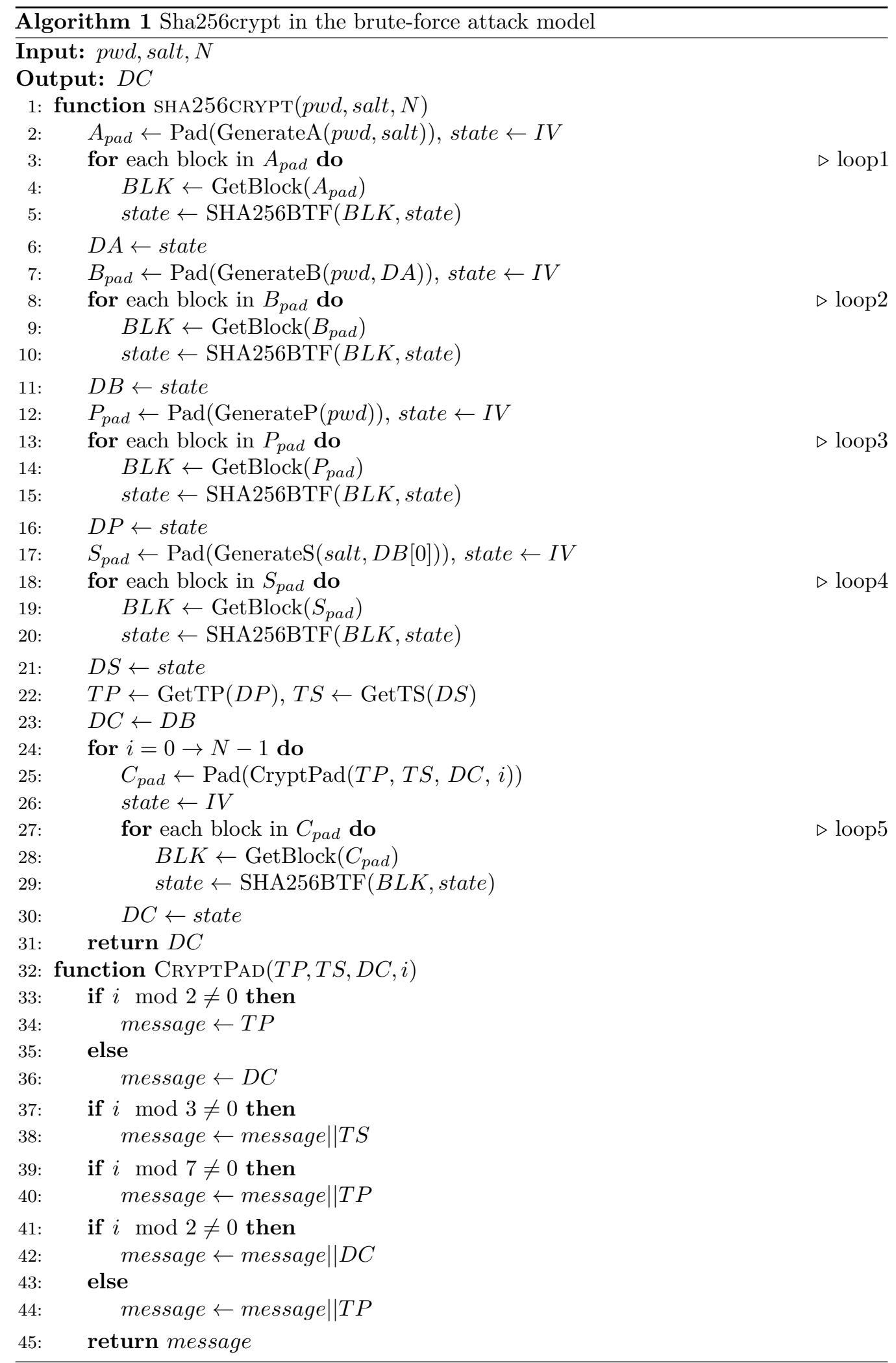


$A, B, P$, and $S$ exactly follow the steps detailed in Section 2.2. Then the state variable is initialized with the $I V$, which is defined in [Dan15]. Inside each round of a loop, GetBlock function is first called to fetch a 64-byte block $(B L K)$ from the input message and then SHA256BTF is called with the BLK and state as its input. The output of SHA256BTF is used to update the state. After the last round of a loop completes, the value of the state is outputted as the digest of the input message. It should be noticed that the message generation and padding operations could be merged into the GetBlock function during the loop, which means there are mainly two types of operations in sha256crypt.

1. Generate a 64-byte block from input sources, which include the bytes in $p w d$, salt, 32-byte digests $(D A, D B, D C, D P, D S)$, temporary variables $(T P, T S)$, 8-byte message length variable $(L)$, constants ('0x80', '0x00').

2. Perform SHA256 block transform function (SHA256BTF) and update the input sources.

We refer the operation 1 as data dispatching and the operation 2 as block transforming. To make an efficient hardware implementation, the cycles needed to compute both operations have to be minimized.

\subsection{Difficulties in Non-blocking Pipelined Accelerator Designs}

A fully-pipelined hash unit outputs one digest per cycle, which means one 64-byte block should also be generated per cycle. However, some features of sha256crypt cause difficulties in the pipelined hash hardware implementation.

The first feature lying in sha256crypt is the data dependency between adjacent SHA256BTFs. An operation can't be executed until the previous operation completes, which stalls the pipeline and increases the average operation cycles.

The second feature is the nonunified execution path. In sha256crypt, the number of rounds in each loop is decided by the number of blocks in each input message $(A, B$, $C, P$, and $S$ ). The variety of the $L P$ and the $L S$ makes the lengths of input message vary, which adversely impacts the schedule of the pipeline. Table 1 gives some examples. There is almost twice the difference between the number of rounds for different passwords, which reduces the efficiency since the fast thread has to wait for the slow thread. As the generation pattern of message $S$ depends on $D B[0]$, which could be considered as a random integer within the interval $[0,255]$, the number of rounds in loop4 might be different even for passwords with the same length. There are 8 cases for the number of rounds in loop5, because the patterns that the CryptPad function uses to generate the message $\mathrm{C}$ is decided by whether the iteration counter $i$ is divisible by 2,3 or 7 , respectively.

Table 1: Number of rounds in each loop for different LP and LS with 5000 iterations.

\begin{tabular}{cccccccc}
\hline LP & LS & loop1 & loop2 & loop3 & loop4 & $\begin{array}{c}\text { loop5 } \\
\text { (each iteration) }\end{array}$ & $\begin{array}{c}\text { loop5 } \\
\text { (5000 iterations) }\end{array}$ \\
\hline 6 & 8 & 1 & 2 & 1 & $3 \sim 35$ & $1,1,1,1,1,1,1,1$ & 5,000 \\
16 & 8 & 1 & 3 & 5 & $3 \sim 35$ & $1,1,2,2,2,2,2,2$ & 9,761 \\
6 & 16 & 1 & 2 & 1 & $5 \sim 68$ & $1,1,1,1,1,1,2,2$ & 7,857 \\
16 & 16 & 1 & 3 & 5 & $5 \sim 68$ & $1,1,2,2,2,2,2,2$ & 9,761 \\
32 & 16 & 2 & 5 & 17 & $5 \sim 68$ & $2,2,2,2,2,2,2,2$ & 10,000 \\
\hline
\end{tabular}

The last feature is the complex block generation pattern. The generation of a block is equivalent to selecting 64 bytes from the source buffers ( $p w d$, salt, $D A, D B$, etc.) and transmitting them to the destination buffer $(B L K)$. Taking loop5 as an example, Figure 4 shows all of the 8 block generation patterns during the $\mathrm{N}$ iterations when $L P$ 
$=6$ bytes and $L S=8$ bytes. Considering the most common case where $L P \leqslant 16$ bytes and $L S=8$ bytes, the total number of bytes in source buffers is 218 ( $p w d$ (16-byte), salt(8-byte), $D X(32$-byte $\times 5), T P(16$-byte), TS(8-byte), $L$ (8-byte), constants(2-byte)). To complete the generation in one cycle, all of the 64 bytes should be fetched simultaneously. If no optimization is applied, sixty-four 218-to-1 multiplexors are needed in hardware implementation, which introduces great overhead to hardware resources and critical path latency.

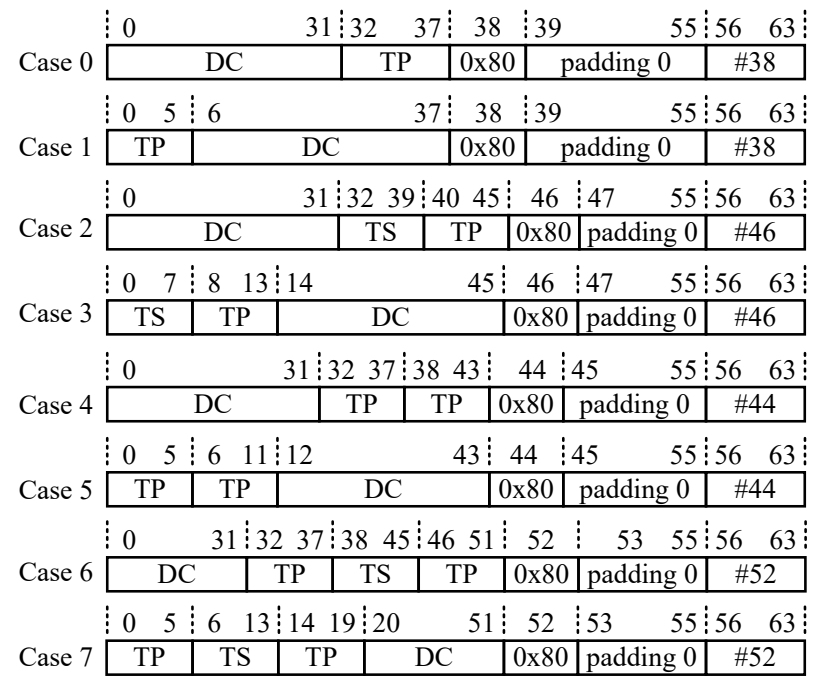

Figure 4: The 8 block generation patterns of loop5 when LP $=6$ bytes and LS $=8$ bytes. The numbers marked on each segment represent its position in the block.

\subsection{Hybrid CPU-FPGA Platforms for Password Recovery}

In the field of password recovery, there's always a trade-off between efficiency and flexibility. Processors with general-purpose architecture, such as CPU and GPU, could easily support all KDFs and provide a flexible user interface. However, they tend to have higher energy consumption than FPGA when performing brute-force attacks. On the other hand, although FPGA achieves better energy efficiency, its poor flexibility limits its application scenarios. Most pure FPGA-based accelerators target the PBKDF2 algorithm, which has a regular and simple structure.

To combine the flexibility and efficiency, hybrid CPU-FPGA platforms, such as the Zynq-7000 SoC series from Xilinx [Xil16] and Stratix 10 SoC series from Intel [Int20], where full-fledged processors are integrated together with configurable logic, can be used to build up hardware password recovery accelerators with both high throughput and low energy consumption [LLD19]. The CPU runs flexible software to make efficient scheduling while the FPGA implements customized hardware logic to provide high performance. Through software and hardware co-design, the designs on FPGA are simplified, thus achieve better efficiency while still maintaining flexibility and low power consumption.

Compared with the discrete CPU-FPGA platforms, the hybrid CPU-FPGA systems like Zynq have additional advantages. First, the smaller chip count simplifies the board-level hardware architecture, cuts down the form factor, and improves the system reliability. Second, the shorter distance between the CPU and the FPGA will cut down the communication delays and of course require less power for the two devices to interact with each other. Last but not least, the hybrid CPU-FPGA device is physically closer to and tightly integrated with its memory, which adds additional overall power saving [CEES14]. 


\section{Overview of System Architecture}

The overall architecture of the proposed accelerator is shown in Figure 5. The system consists of a CPU, a secure digital memory card (SD card), and an FPGA with several sha256crypt accelerating cores. The CPU and the FPGA are integrated on the same system on chip (SoC) and linked with AXI bus. The SD card's functionality is to store the bitstream files used to configure the FPGA.

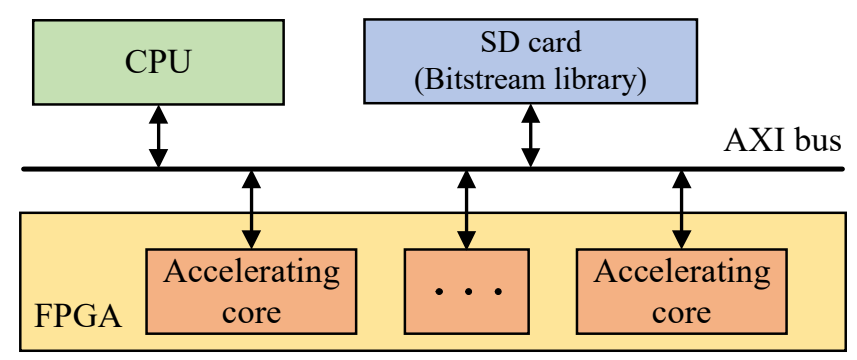

Figure 5: Overall architecture of the proposed password recovery accelerator.

To provide a user-friendly interface and an efficient task management, a password recovery framework is deployed on the CPU. The framework has the same front end with the famous password recovery tool Hashcat [has20], which supports flexible attack modes (dictionary, mask and rule, etc.) that allow users to accurately specify the password searching space. The back end of the framework handles the task arrangement between the CPU and the FPGA.

Figure 6 shows the flow chart of the sha256crypt password recovery. First, the password hash is parsed to get the salt, the iteration number $(N)$, and the decoded check value. Then, for each possible value of $D B[0]$ in Phase III-3, the CPU generates a message $S$ and calculates its digest $D S$. This procedure is named as look-ahead execution. Next, a group of passwords with the same length is generated from dictionaries or masks specified by the user. The FPGA will be reconfigured according to the length of the password if necessary. After that, the salt, $N$, pre-calculated $D S \mathrm{~s}$, and the passwords are transmitted to the accelerating cores on the FPGA through the AXI bus. When the accelerating cores complete the sha256crypt computation, the results are sent back to the CPU. Finally, the CPU compares these results with the check value. If a match is found, the correct password will be outputted. Otherwise, the process above will be repeated until the correct password is found or the searching space is exhausted.

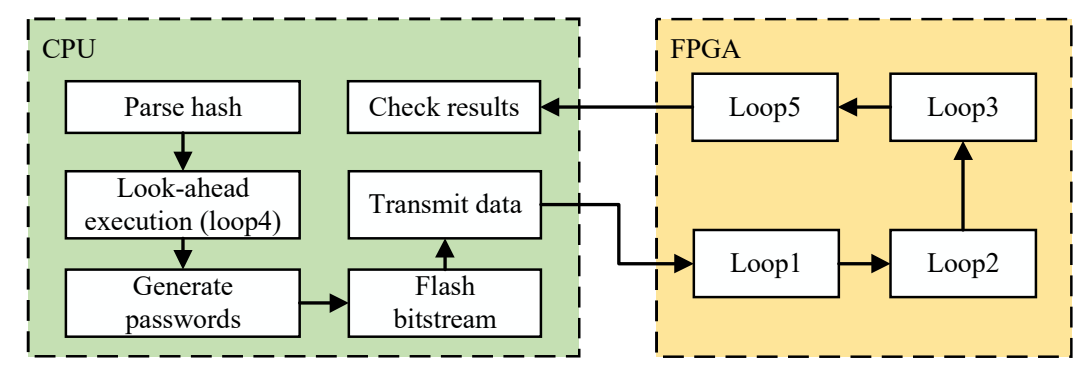

Figure 6: Flow chart of sha256crypt password recovery on the proposed accelerator.

The architecture of the accelerating cores is shown in Figure 7. There are three components namely the data buffers, the data dispatch unit, and the block transform unit. The data buffers store the input sources, which include $p w d$, salt, $D A, D B$, etc. The data dispatch unit selects 64 bytes from the input sources to generate blocks under the 
control of the finite state machine (FSM). Then the block transform unit takes the blocks generated by the data dispatch unit and performs SHA256BTF to generate digests. For the advantage of high throughput, the pipeline technique is applied to the block transform unit. As the technique for efficient SHA256 implementations [MCMM06, CKSV08, RVS15] has been well studied, it is not the focus of our paper. Finally, these digests are sent to the state buffer or data buffers to update the states or input sources. Operations above finish one round of a loop. When the last round of all the loops completes, the results are transmitted back to the CPU for validating.

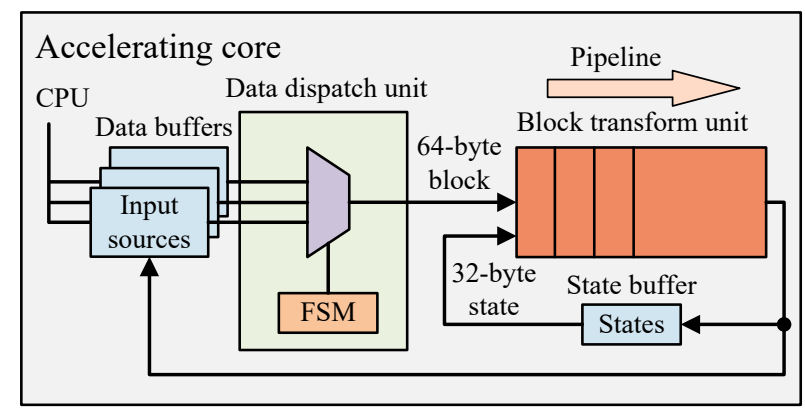

Figure 7: Architecture of the proposed accelerating core.

Figure 8 shows the task arrangement between the CPU and the FPGA. The task of checking results on the CPU and the acceleration on the FPGA are parallelized to improve the efficiency. As it requires a substantial modification to Hashcat's source code but gains little improvement, the task of generating passwords is not parallelized. The communication overhead comes from the transmission of the passwords and results.

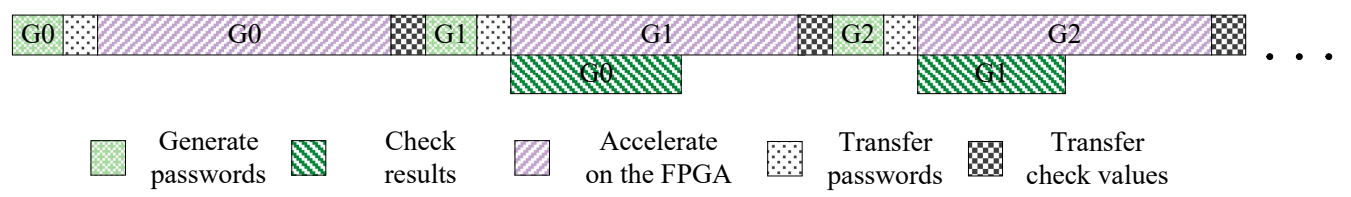

Figure 8: Task arrangement between the CPU and the FPGA. The task of checking results in the group 0 (G0) and the FPGA acceleration in the group 1 (G1) are processed concurrently, which is the same for subsequent groups like the group 1 and group 2 (G2).

\section{Hardware Implementation}

Hardware implementation details of our accelerator are provided in this section. Section 4.1 discusses the group scheduling technique to eliminate data hazard of the pipelined block transform unit. Section 4.2 introduces the look-ahead execution technique to unify the execution paths. Section 4.3 introduces the efficient design of the data dispatch unit using the data path pruning and the spatial-temporal multiplexing techniques. Section 4.4 shows the detailed architecture of the accelerating core with all these techniques put together.

\subsection{Group Scheduling}

As mentioned in Section 2.4, during the loop process, data dependency exists between two adjacent block transform operations. As a result, each block has to wait $M$ cycles 
until the processing of its previous block is finished, where $M$ is the stage of the pipeline. For the convenience of hardware design, $M$ is set to 64 in our implementation. We use the utilization rate of the block transform unit to quantify the efficiency of the scheduling scheme, which is defined as follows:

$$
\text { Utilization rate }=\frac{\text { actual block throughput }}{\text { designed block throughput }}
$$

Since the designed block throughput is one block per cycle, the utilization rate also equals to the average number of blocks processed per cycle. If the data dependency is not solved, the utilization rate of the block transform unit could only reach $1 / 64$.

To eliminate the data dependency and improve the utilization rate of the pipelined block transform unit, the group scheduling technique proposed in [LLD19] is applied. As there is no data dependency between the processing of different passwords, a group of $G$ passwords can and shall be stored and processed together. We assume that all the passwords in a group have the same number of blocks to process, which can be guaranteed by applying the look-ahead execution technique.

Figure 9 gives the comparison between pipelines with and without the group scheduling. By applying the group scheduling, the full-filled pipeline completes one block transform operation per cycle. Taking the overhead of the pipeline full-filling into consideration, the utilization rate of the block transform unit will be $\mathrm{G} /(\mathrm{G}+64)$. To maximize the utilization rate of the block transform unit, the group size $G$ should be maximized under the limitation of available BRAM resources.

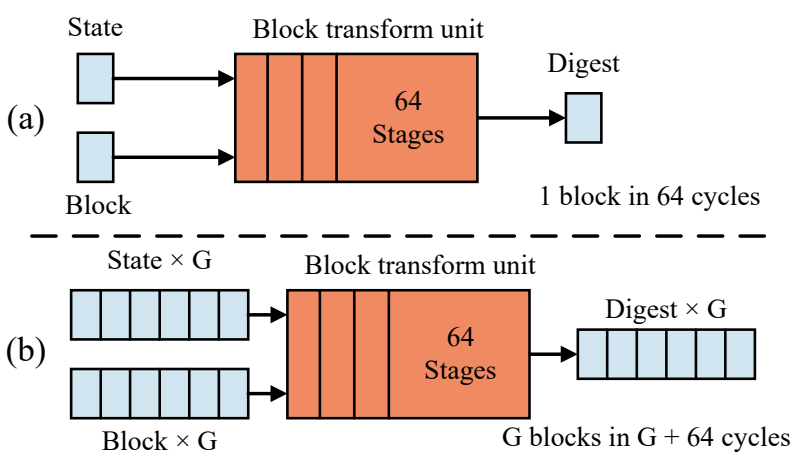

Figure 9: (a) Pipeline without group scheduling. (b) Pipeline with group scheduling.

\subsection{Look-ahead Execution}

Section 2.4 has shown that different passwords and salts lead to different execution paths of sha256crypt. This feature brings difficulties in group scheduling, since the threads in the same group may have different numbers of blocks to process. As the salt remains unchanged during the password recovery, the uncertainty of execution paths comes from two aspects: the variation of password length and the unpredictable value of $D B[0]$ in Phase III-3.

Variation of password length can be eliminated by sorting passwords by length when generating passwords on the CPU. Then passwords with the same length are grouped and transferred to the accelerating core.

To remove the uncertainty caused by the unpredictable value of $D B[0]$, we propose the look-ahead execution technique based on the following observations. First, there is only one salt during the cracking of one password hash. Second, $D B[0]$ has a range of 0 to 255 . 
As a result, there are only 256 possible values of $D S$ in total and we can reuse them for all passwords.

To this end, the calculation of $D S$ (loop4) is mapped to the CPU. As shown in Figure 10, for each possible value of $D B[0]$, a $D S$ is calculated and stored in the LAE (Look-Ahead Execution) buffer of the accelerating core. During the processing of the accelerating core, once the calculation of each $D B$ completes, $D B[0]$ is used to select corresponding $D S$ from $256 D S \mathrm{~s}$ in the LAE buffer. The results are stored in the $D S$ buffer. With the look-ahead execution technique, the value of $D S$ is decided once the value of $D B$ is calculated. Thus, all the passwords in the same group will follow the same execution path.

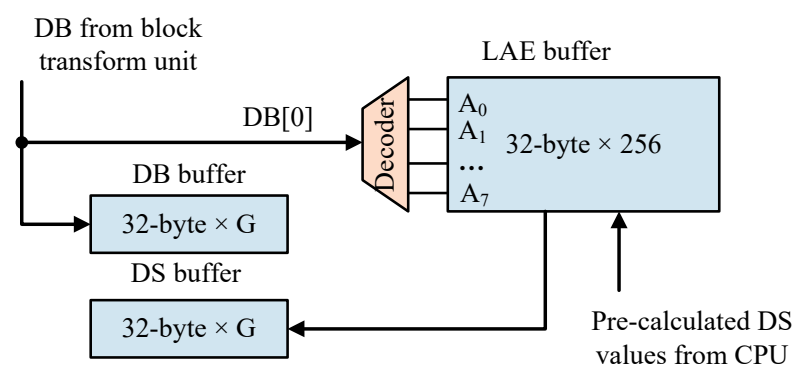

Figure 10: Schematic of the look-ahead execution technique.

\subsection{Efficient Data Dispatch Unit Design}

An efficient design of the data dispatch unit with low overhead is necessary on a resourcestrapped FPGA. Figure 11 illustrates the data path between the input sources and the output block, where 64 multiplexors are used to select 64 bytes from the input sources. Each multiplexor is controlled by a control signal generated by the FSM.

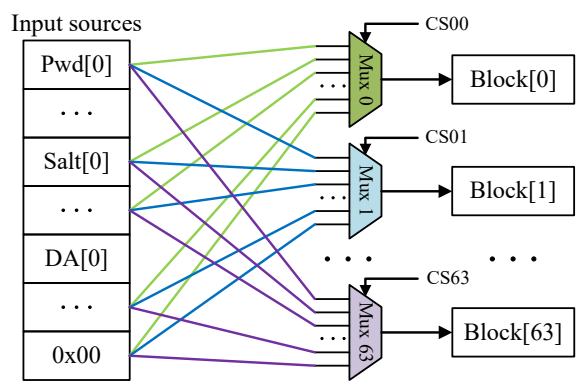

Figure 11: Data path between the input sources and the output block.

To describe the behavior of the FSM, we first define a state group for each loop in sha256crypt. As shown in Figure 12, a state group consists of several states. Each state corresponds to a round of the loop. For example, when $L P=6$ bytes and $L S=8$ bytes, state groups for loop1 and loop3 (SG1, SG3) both have one state, while the state group for loop2 (SG2) has two states. As loop4 is mapped to the CPU by look-ahead execution, there is no state group assigned to it. For loop5, we define 42 state groups, since its message generation pattern changes with a period of 42 during the iterations, which is the least common multiple of 2, 3, and 7. According to Table 1, all state groups for loop5 have one state. In this case, a total of 46 states are needed. The state $\mathrm{S}_{\mathrm{E}}$ is added as the end state.

The numbers of states needed for each password length are listed in Table 2. A total of 815 states are needed to support $L P$ going from 6 to 16 bytes when $L S=8$ bytes. 


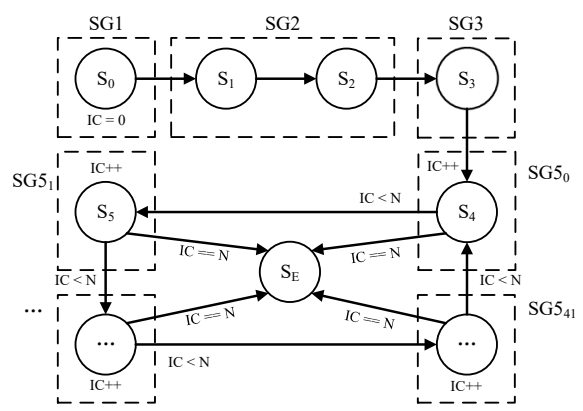

Figure 12: State diagram of the finite state machine for $L P=6$ bytes and $L S=8$ bytes. The $I C$ (iteration counter) is set to 0 at $\mathrm{S}_{0}$. Each time when the FSM leaves SG5, the $I C$ is increased by one. Once the $I C$ equals the iteration number $N$, the FSM transits to the end state $\mathrm{S}_{\mathrm{E}}$.

Table 2: Number of states needed in FSM for LP from 6 to 16 bytes when LS $=8$ bytes.

\begin{tabular}{cccccccccccc}
\hline LP & 6 & 7 & 8 & 9 & 10 & 11 & 12 & 13 & 14 & 15 & 16 \\
\hline \# of states & 46 & 46 & 71 & 71 & 71 & 73 & 85 & 85 & 88 & 88 & 90 \\
\hline
\end{tabular}

Design of the FSM is shown in Figure 13. Benefiting from the unified execution path, the FSM can be simply implemented with a BRAM-based look-up-table. The content in the look-up-table has 3 segments, which are the next state, the control signals, and the end of loop5 flag (EOL). At each state, the value in the state counter is used as the address to access the content in the look-up-table. Then the state counter is updated with the next state. The control signals from CS00 to CS63 are used to control the multiplexors of the data path. The EOL flag is used to indicate whether a state group for loop5 is finished. If the EOL is 1, the iteration counter should increase by one.

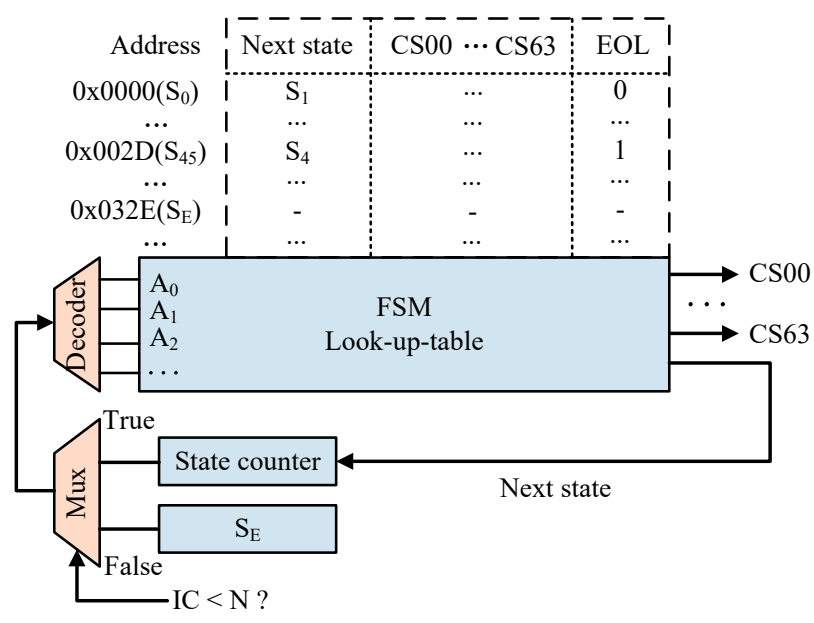

Figure 13: Design of the finite state machine.

We adopt two factors to quantify the overhead of the data dispatch unit. The first is the number of connections between the input sources and the output block. The number of connections will influence the LUT resources that are used to implement multiplexors. Another factor is the size of the look-up-table, which affects the usage of BRAM resources on FPGA.

Considering a simple and intuitive design where each multiplexor is connected directly 
with all input sources, as mentioned in Section 2.4, the number of connections for each multiplexor is 218 . The total number of connections would be $218 \times 64=13,952$, which costs 39,901 LUTs (about 50\% of the total LUTs on our experimental platform). Meanwhile, for a 218-to-1 multiplexor, at least 8 bits of control signals are needed. Including 10 bits for the next state segment and 1 bit for the EOL segment, the size of storage needed for each state is $8 \times 64+10+1=523$ bits. The total size of the look-up-table will be $523 \times 815=$ 426,245 bits (about $52 \mathrm{KiB}$ ). To reduce the overhead of the data dispatch unit, we propose data path pruning and spatial-temporal multiplexing techniques.

\section{A. Data path pruning}

The data path pruning technique is based on the following observations. First, different input sources could share the same storage resources. For example, $D B$ and $D C$ could share the same buffer since they will not be used at the same time. Second, for a specific byte in the block, its candidates only account for a part of all input sources, which means a multiplexor doesn't have to connect with all input sources.

Exploiting these observations, we reuse the buffers for multiple variables, which is listed in Table 3. Then, we customize the size of each multiplexor, so that only the possible candidates are connected to the inputs of a multiplexor. Figure 14(a) and Figure 14(b) show the number of connections before and after applying data path pruning. After pruning, the total number of bytes in input sources is decreased and each multiplexor has fewer connections. Therefore, the total number of connections is reduced from 13,952 to 3,086 .

Table 3: Relationship between the variables and the buffers they use.

\begin{tabular}{cccccc}
\hline Buffer name & pwd & salt & DA & DB & DS \\
\hline Variable name & pwd, TP & salt & DA, DP, DC & DB, DC & DS, TS \\
\hline
\end{tabular}

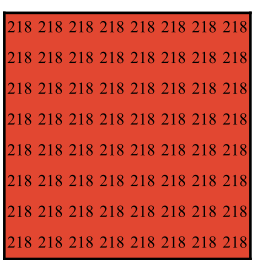

(a) Unoptimized

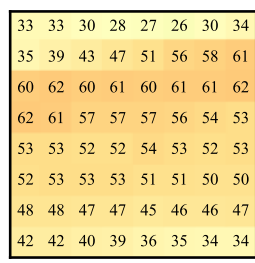

(b) After pruning

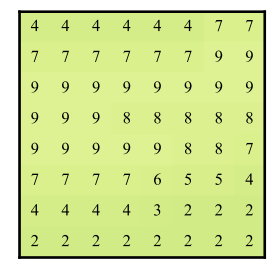

(c) After pruning and spatial-temporal multiplexing

Figure 14: Data path hotmap. Each grid represents a byte in the block. The number marked on the grid represents the number of connections for the corresponding multiplexor.

\section{B. Spatial-temporal multiplexing}

It should be noticed that the variation of password lengths also makes a great contribution to the complexity of the data path. Figure 14(c) shows the number of connections actually used when the length of the password is fixed to 6 bytes. In this case, the number of active connections is reduced to 394. This fact drives us to explore the temporal locality of the data path and propose the spatial-temporal multiplexing technique, which leverages the reconfigurability of the FPGA.

For password length from 6 bytes to 16 bytes, we prune the data path and generate a bitstream file for each length. Passwords with the same length are grouped and sent to the FPGA for processing. Every time the length of passwords changes, the FPGA is reconfigured with corresponding bitstream file. The software running on the CPU will sort 
the passwords by length to avoid frequent reconfiguration. With this technique, the whole data path is separated and distributed into different bitstream files.

As the number of connections is reduced, each multiplexor needs fewer bits of control signals. What's more, since each design only needs to store the states of a specific password length, the total storage overhead can be further reduced. In the case where $L P=6$ bytes and $L S=8$ bytes, each control signal costs 4 bits and there are 47 states need to be stored. As a result, the storage overhead of the look-up-table is $47 \times(4 \times 64+6+1)=12,361$ bits, which is about $3 \%$ of the unoptimized design.

\subsection{Putting It All Together}

Figure 15 shows the hardware implementation details of the accelerating core. The implementation provided in Figure 15 is specialized for passwords of 6 bytes and salts of 8 bytes due to the spatial-temporal multiplexing technique. For other combinations of passwords and salts, the proposed architecture is also adaptable by adjusting the connection of the data path and the size of the pwd and salt buffer.

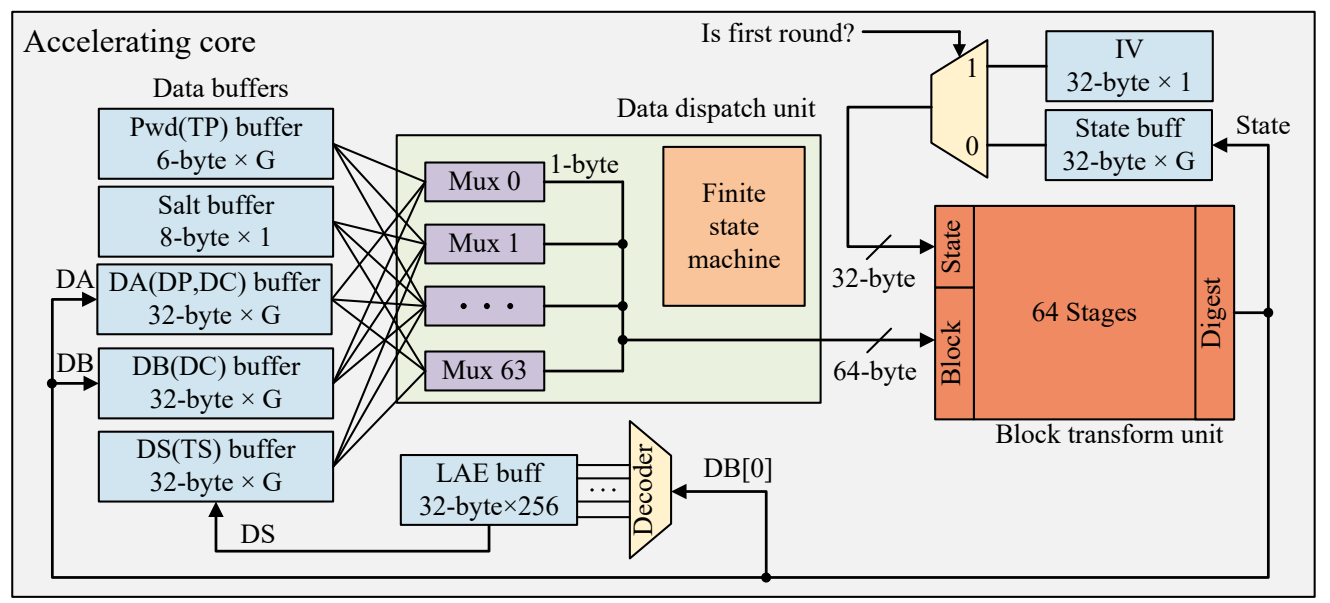

Figure 15: The detailed architecture of the accelerating core.

The data buffers include the pwd, salt, $D A, D B$, and $D S$ buffers, which are used as the input sources. The LAE buffer receives the pre-calculated DSs generated by the look-ahead execution on the CPU. The state buffer stores the intermediate digests that are used as the states in SHA256 calculations, such as the $\mathrm{D}_{1}, \mathrm{D}_{2}, \ldots$, and $\mathrm{D}_{\mathrm{N}-1}$ in Figure 3 . In order to apply the group scheduling technique, all buffers are organized in a 2-dimensional manner. The first dimension is the size of the variable and the second dimension is the size of the group, which is denoted with $G$. The salt buffer has a group size of one, since all passwords share the same salt. As the 64-byte block and the 32-byte state needed by SHA256BTF have to be fetched simultaneously, these buffers are completely partitioned at the first dimension.

Once the configuration of the FPGA is complete, all the accelerating cores are ready to work. Each core processes a group of passwords with the following steps:

Loop1: The pwd and salt buffer receive the passwords and salt from the CPU. Then they are used as the input sources to calculate $D A$, which is then stored in the $D A$ buffer.

Loop2 and loop4: The $p w d$ buffer and $D A$ buffer are used as the input sources to calculate $D B$, which is then stored in the $D B$ buffer. Meanwhile, $D S$ is selected from the LAE buffer and stored in the $D S(T S)$ buffer. 
Loop3: The $p w d$ buffer is used as the input sources to calculate $D P$, which is then stored in the $D P$ buffer. Then the first \#LP byte of $D P$ is copied into the $T P$ buffer.

Loop5: The $T P, T S$, and $D B$ buffers are used as the input sources to calculate $D C$, which is then stored in the $D A$ buffer. In the following iterations, the $D A$ and $D B$ buffers are alternatively used as the input sources and output buffer for $D C$.

At each round of a loop, the data dispatch unit switches to the corresponding block generation pattern. One block is generated and sent to the block transform unit every cycle. The block transform unit performs SHA256BTF on these blocks and outputs the digests. If the current round is not the last round of a loop, the digests will be stored in the state buffer as the intermediate states. Otherwise, they will be stored in the data buffers. After the processing of all $G$ blocks in a group is finished, the data dispatch unit switches to the next block generation pattern to start a new round. When all the rounds of all the loops are finished, the final digests of $D C$ are sent back to the CPU to compare with the password check value.

\section{Evaluation}

The performance and energy efficiency of our accelerator are evaluated on a Xilinx Zynq7000 XC7Z030-3 SoC, which contains two ARM Cortex-A9 processors working at 666 $\mathrm{MHz}$ and a 7 series programmable logic. Two sha256crypt accelerating cores working at a maximum frequency of $220 \mathrm{MHz}$ are placed on the FPGA. The accelerating cores are synthesized and implemented with the Xilinx Vivado 2016.3 toolset. To justify the proposed designs, the implementations are compared with the state-of-the-art password recovery tools Hashcat [has20] and John-the-Ripper (JtR) [Mag18]. The proposed accelerator is also evaluated on a 16-node cluster which consists of the same Zynq devices, as is shown in Appendix A.

\subsection{System Performance}

The system performance of the proposed accelerator is shown in Table 4. As the number of blocks in sha256crypt increases with the length of the password, the performance statistics for length from 6 bytes to 16 bytes are all reported. We use the number of passwords that the system can check per second as the indicator of speed. The power consumption is measured at the DC power supply of $5 \mathrm{~V}$. The energy efficiency indicates the energy cost for each password, which is calculated by speed/power. The proposed accelerator achieves a speed of $82,023 \mathrm{pwd} / \mathrm{s}$ when $L P=6$ bytes. As the $L P$ increases to 16 bytes, the speed drops to $42,658 \mathrm{pwd} / \mathrm{s}$ since the number of blocks for each password increases. The power consumption of the proposed accelerator is about 15 Watts.

Table 4: System performance of the proposed accelerator

\begin{tabular}{cccccccccccc}
\hline LP & 6 & 7 & 8 & 9 & 10 & 11 & 12 & 13 & 14 & 15 & 16 \\
\hline \# of blocks & 5,023 & 5,023 & 7,881 & 7,881 & 7,881 & 7,883 & 9,311 & 9,311 & 9,312 & 9,312 & 9,789 \\
Speed (pwd/s) & 82,023 & 82,016 & 52,992 & 52,844 & 52,834 & 52,881 & 44,882 & 44,686 & 44,799 & 44,797 & 42,658 \\
Power (Watt) & 14.78 & 15.13 & 13.53 & 13.68 & 13.88 & 13.78 & 13.28 & 14.98 & 15.03 & 14.93 & 15.03 \\
Energy efficiency & 5,550 & 5,421 & 3,918 & 3,867 & 3,807 & 3,838 & 3,381 & 2,983 & 2,981 & 3,001 & 2,838 \\
$\quad($ pwd/J) & & & & & & & & & & & \\
\hline
\end{tabular}

\subsection{Analysis of the Performance Overhead}

We use the utilization rate of the block transform unit as the figure of merit to evaluate the performance overhead, which mainly comes from the pipeline full-filling, the password generation, and the communication delay between the CPU and FPGA. 
The detailed time cost for each task when $L P=6$ bytes is listed in Table 5 . In our implementation, the group size $G$ is set to 2048 to maximize the utilization rate under the limitation of available BRAM resources. According to Table 1, each password has 5,004 blocks to be processed on the FPGA. As the time of checking results on the CPU is hidden behind the time of acceleration on the FPGA, the processing of a password group costs about $49,444 \mu \mathrm{s}$. The measured block throughput is about $4.15 \times 10^{8}$ block $/ \mathrm{s}$. Table 6 compares the theoretical utilization rate and the measured utilization rate. The proposed accelerator reaches an average utilization rate of $94.3 \%$. If we only consider the time for the acceleration on the FPGA, the equivalent block throughput will be $4.25 \times 10^{8}$ block $/ \mathrm{s}$, which is close to the theoretical value.

Table 5: Time cost for a group of 2048 passwords when LP $=6$ bytes.

\begin{tabular}{|c|c|c|c|}
\hline Task & Blocks & Time cost $(\mu \mathrm{s})$ & $\begin{array}{c}\text { Throughput } \\
\text { (block/s) }\end{array}$ \\
\hline Generate passwords & & 572 & - \\
\hline Transfer passwords & & 310 & - \\
\hline $\begin{array}{l}\text { Accelerate on the FPGA } \\
\text { (Check results) }\end{array}$ & $2048 \times 5004 \times 2$ & $\begin{array}{c}48,230 \\
(18,339)\end{array}$ & $4.25 \times 10^{8}$ \\
\hline Transfer check values & & 331 & - \\
\hline Total & & 49,444 & $4.15 \times 10^{8}$ \\
\hline
\end{tabular}

Table 6: Utilization rate of the block transform unit (two cores).

\begin{tabular}{ccccc}
\hline & \# of Blocks & Time $(\mathrm{s})$ & $\begin{array}{c}\text { Throughput } \\
(\text { block } / \mathrm{s})\end{array}$ & Utilization rate \\
\hline Theoretical & $2048 \times 2$ & $(2048+64) \div\left(2.20 \times 10^{8}\right)$ & $4.27 \times 10^{8}$ & $97.0 \%$ \\
Measured & $2048 \times 5004 \times 2$ & $49444 \times 10^{-6}$ & $4.15 \times 10^{8}$ & $94.3 \%$ \\
\hline
\end{tabular}

The time for the look-ahead execution on the CPU is negligible since it only executes once. The time of each reconfiguration is $243 \mathrm{~ms}$. The overhead introduced by reconfiguration is determined by the frequency of reconfiguration. For some small dictionaries where the FPGA needs to be reconfigured every 10 groups, the average time overhead for each group is $24.3 \mathrm{~ms}$, which will reduce the performance for about $33.5 \%$. In some other cases where the mask mode is applied, the FPGA is reconfigured every 10,000 groups or more, which will bring down the time overhead to $24 \mu$ s or less, barely noticeable.

\subsection{Analysis of the Data Path Overhead}

As mentioned in Section 4.3, the number of connections in the data path affects the hardware resource overhead of the data dispatch unit. Figure 16 shows the number of connections and corresponding LUT usage of the data dispatch unit for different passwords. Compared with the unoptimized design, which uses 39,901 LUTs, the designs with the data path pruning and spatial-temporal multiplexing techniques occupy about 6,000 to 10,000 LUTs. The result also implies that as the number of connections changes with the password length, the LUT usage of the data dispatch unit changes with the same trend. In other words, the number of connections is a good indicator of the LUT usage of the data dispatch unit. 


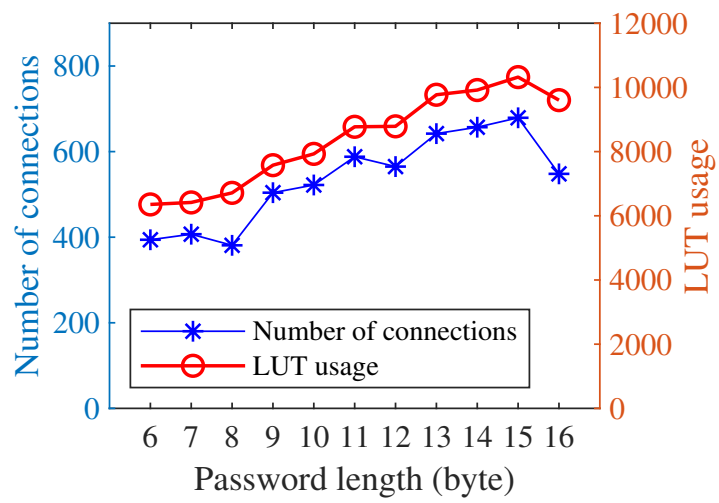

Figure 16: Number of connections in the data path and LUT usage for the data dispatch unit.

\subsection{Comparison with Other Schemes}

\subsubsection{Comparison with FPGA-based non-pipelined implementation}

The sha256crypt implementation of John-the-Ripper (JtR) [Mag18] places 23 computing units per FPGA for acceleration, which is detailed in Appendix B. Each computing unit contains a bespoke CPU to generate and dispatch message blocks and 3 non-pipelined SHA256 cores to finish the SHA256 block transforming. Each SHA256 core costs 72 cycles on average to process one block.

To make a fair comparison, we reproduce the sha256crypt implementation of JtR on the Zynq-7000 XC7Z030-3 platform. The system speed, energy efficiency, and resource efficiency are used as the figures of merit, which are shown in Table 7. Both the implementation of JtR and our accelerator are tested under the wordlist mode. As the number of blocks for different passwords and salts varies, the energy efficiency and resource efficiency are evaluated by block/J and block/s/LUT.

Comparing both designs at their respective maximum frequencies, our accelerator achieves $1.74 \times$ block throughput and $1.64 \times$ energy efficiency over JtR. The LUTs used as computing logic in JtR account for about $66.1 \%$, while the proposed accelerator has a proportion of $88.5 \%$, which means more hardware resources in our design are used to computing other than block generation and controlling. The resource efficiency of our accelerator is $1.69 \times$ better than JtR.

Table 7: Comparison with JtR

\begin{tabular}{lcc}
\hline & JtR (300 MHz) & This work (220 MHz) \\
\hline \multicolumn{3}{c}{ Speed and power (pwd/s // Watt) } \\
\hline $\mathrm{LP}=8, \quad \mathrm{LS}=8, \quad 7,881$ blocks & $29,810 / / 12.75$ & $52,992 / / 13.53$ \\
$\mathrm{LP}=16, \mathrm{LS}=8, \quad 9,789$ blocks & $25,174 / / 13.55$ & $42,658 / / 15.03$ \\
$\mathrm{LP}=8, \quad \mathrm{LS}=16,8,375$ blocks & $28,088 / / 13.15$ & $50,046 / / 12.52$ \\
$\mathrm{LP}=16, \mathrm{LS}=16,9,807$ blocks & $25,165 / / 13.65$ & $42,638 / / 15.69$ \\
\hline \multicolumn{4}{c}{ Energy efficiency } \\
\hline Block throughput(block/s) & $2.41 \times 10^{8}(1 \times)$ & $4.18 \times 10^{8}(1.74 \times)$ \\
Energy efficiency (block/J) & $1.81 \times 10^{7}(1 \times)$ & $2.97 \times 10^{7}(1.64 \times)$ \\
\hline \multicolumn{4}{c}{ Resource efficiency } \\
\hline Average LUT usage & 54,917 \\
LUTs as computing logic & $36,294(66.1 \%)$ & $49,927(88.5 \%)$ \\
Resource efficiency (block/s/LUT) & $4,386(1 \times)$ & $7,409(1.69 \times)$ \\
\hline
\end{tabular}




\subsubsection{Comparison with GPU}

Table 8 shows the comparison of the performance and energy efficiency between our accelerator and Hashcat(v5.1.0) [has20] running on an NVIDIA GTX1080Ti GPU. Beside the normal mode, Hashcat provides an optimized mode which has better performance for passwords shorter than 16 bytes. We choose the optimized mode as the baseline. The result has shown that the proposed accelerator achieves $2.54 \times$ improvement in energy efficiency than Hashcat running on GTX1080Ti. Although the block throughput of a single node is only $0.15 \times$ of Hashcat, when we test our design on the 16-node cluster, the block throughput is $2.41 \times$ better than Hashcat. The total power of the cluster is estimated to be 16 times of the power consumed by a single node.

Table 8: Comparison with Hashcat running on a GTX1080Ti for sha256crypt.

\begin{tabular}{lcccc}
\hline & $\begin{array}{c}\text { Hashcat } \\
\text { normal }\end{array}$ & $\begin{array}{c}\text { Hashcat } \\
\text { optimized } \\
\end{array}$ & $\begin{array}{c}\text { This work } \\
\text { single node }\end{array}$ & $\begin{array}{c}\text { This work } \\
\text { cluster }\end{array}$ \\
\hline $\mathrm{LP}=8, \quad \mathrm{LS}=8, \quad 7,881$ blocks & $93,264 / / 215$ & $355,300 / / 236$ & $52,992 / / 13.53$ & $847,347 / / 216.43$ \\
$\mathrm{LP}=16, \mathrm{LS}=8, \quad 9,789$ blocks & $74,266 / / 199$ & Unsupported & $42,658 / / 15.03$ & $682,498 / / 240.48$ \\
$\mathrm{LP}=8, \quad \mathrm{LS}=16,8,375$ blocks & $80,485 / / 197$ & $327,500 / / 239$ & $50,046 / / 12.52$ & $798,590 / / 200.40$ \\
$\mathrm{LP}=16, \mathrm{LS}=16,9,807$ blocks & $75,380 / / 204$ & Unsupported & $42,638 / / 15.69$ & $681,004 / / 251.10$ \\
\hline \multicolumn{5}{c}{ Energy efficiency } \\
\hline Block throughput (block/s) & $7.19 \times 10^{8}(0.26 \times)$ & $2.77 \times 10^{9}(1 \times)$ & $4.18 \times 10^{8}(0.15 \times)$ & $6.68 \times 10^{9}(2.41 \times)$ \\
Energy efficiency (block/J) & $3.53 \times 10^{6}(0.30 \times)$ & $1.17 \times 10^{7}(1 \times)$ & $2.97 \times 10^{7}(2.54 \times)$ & $2.97 \times 10^{7}(2.54 \times)$ \\
\hline
\end{tabular}

To evaluate the efficiency of the architectures between GPU and our accelerator, we use the utilization rate of the computing unit as the figure of merit, which is calculated by the measured block throughput divided by the maximum block throughput. The maximum block throughput of our accelerator is $4.40 \times 10^{8}$ block/s. The maximum block throughput of GTX1080Ti is reported by Hashcat's SHA256 benchmark, which is $4.42 \times 10^{9}$ block/s. The result shows that GTX1080Ti only utilizes $62.7 \%$ of its maximum ability, while our accelerator utilizes $94.5 \%$ of its maximum ability. The proposed architecture of our accelerator makes better use of computing units than the general-purpose architecture of GPU.

\subsection{Adapting to sha512crypt}

To validate the adaptability of the techniques proposed in this paper, we also implement sha512crypt on the same platform. Since SHA512 costs more hardware resource than SHA256, only one sha512crypt accelerating core is placed, which also works at $220 \mathrm{MHz}$. The performance comparison with Hashcat is shown in Table 9. The proposed accelerator achieves $2.39 \times$ energy efficiency over Hashcat running on an NVIDIA GTX1080Ti GPU.

\subsection{Disussion about Cost}

Cost of the password recovery mainly comes from two areas, the BOM (bill of materials) and the energy. The BOM cost is the one-time cost to build the hardware while the energy cost is the recurring cost to run the hardware. Assuming that two password recovery solutions have the same speed, the total cost increases linearly with time. Taking the data from Table 8, a GTX1080Ti has the same speed with about 7 nodes of the proposed accelerator while it needs $2.54 \times$ more electricity. The BOM costs of a GTX1080Ti and each node of our accelerator are about 1,000 USD and 450 USD, respectively. Assuming that the electricity price is $0.15 \mathrm{USD} / \mathrm{kWh}$, we can find the dollar figure for the energy consumption is $0.036 \mathrm{USD} / \mathrm{h}$ for a GTX1080Ti $(240 \mathrm{~W})$ and it would be $0.014 \mathrm{USD} / \mathrm{h}$ for 
Table 9: Comparison with Hashcat running on a GTX1080Ti for sha512crypt.

\begin{tabular}{lcccc}
\hline & $\begin{array}{c}\text { Hashcat } \\
\text { normal }\end{array}$ & $\begin{array}{c}\text { Hashcat } \\
\text { optimized }\end{array}$ & $\begin{array}{c}\text { This work } \\
\text { single node }\end{array}$ & $\begin{array}{c}\text { This work } \\
\text { cluster }\end{array}$ \\
\hline & \multicolumn{5}{c}{ Speed and power $($ pwd/s // Watt) } \\
\hline $\mathrm{LP}=8, \mathrm{LS}=8, \quad 5,014$ blocks & $26,384 / / 175$ & $192,900 / / 245$ & $41,086 / / 18.91$ & $667,774 / / 302.60$ \\
$\mathrm{LP}=16, \mathrm{LS}=8,5,016$ blocks & $26,407 / / 184$ & $192,100 / / 241$ & $40,855 / / 21.23$ & $652,368 / / 339.63$ \\
$\mathrm{LP}=8, \quad \mathrm{LS}=16,5,023$ blocks & $26,304 / / 182$ & $190,300 / / 242$ & $41,083 / / 18.91$ & $656,388 / / 302.60$ \\
$\mathrm{LP}=16, \mathrm{LS}=16,7,882$ blocks & $24,642 / / 193$ & $188,900 / / 243$ & $26,317 / / 17.66$ & $419,909 / / 282.48$ \\
\hline & \multicolumn{5}{c}{ Energy efficiency } \\
\hline Block throughput (block/s) & $1.48 \times 10^{8}(0.14 \times)$ & $1.09 \times 10^{9}(1 \times)$ & $2.06 \times 10^{8}(0.19 \times)$ & $3.29 \times 10^{9}(3.02 \times)$ \\
Energy efficiency (block/J) & $8.02 \times 10^{5}(0.18 \times)$ & $4.51 \times 10^{6}(1 \times)$ & $1.08 \times 10^{7}(2.39 \times)$ & $1.08 \times 10^{7}(2.39 \times)$ \\
\hline
\end{tabular}

our accelerator ( 7 nodes, $94 \mathrm{~W}$ ). It will take 11 years of operation time until our accelerator beats the other party in total cost, which seems unacceptable at first sight.

However, as there are a lot of factors that affect the BOM cost and energy cost, this conclusion may change for different cases. For example, if a company in Germany purchases Zynq devices in a large quantity to receive a deeply discount price of 200 USD and the electricity price is $0.35 \mathrm{USD} / \mathrm{kWh}$, it will only take 327 days, less than one year, to win over the GTX1080Ti-based solutions in a dollar sense.

\section{Conclusion and Future Work}

This paper has proposed an efficient sha256crypt password recovery accelerator based on the pipelined SHA256 unit. By analyzing the sha256crypt algorithm, we find that pipelining is particularly suitable for accelerating the computation of KDFs which have a structure consisting of cascaded message blocks. Based on this important observation, we propose a pipeline-based architecture. To overcome the difficulties in pipelined hardware implementation and reduce the hardware resource overhead, several techniques are proposed, such as group scheduling, look-ahead execution, data path pruning, and spatial-temporal multiplexing. With these techniques, our accelerator achieves $2.54 \times$ improvement on energy efficiency compared with the state-of-the-art password recovery tool Hashcat running on an NVIDIA GTX1080Ti GPU. Compared with the pure FPGA-based accelerator in JtR, our accelerator achieves $1.64 \times$ energy efficiency and $1.69 \times$ resource efficiency. Our study shows that the unified execution path and simple data flow are the keys to high efficiency, and high efficiency implies both high performance and low overhead for the password recovery accelerators.

Without loss of generality, the techniques proposed in this paper can also be applied to the KDFs that have similar features, such as sha512crypt and md5crypt. We appreciate the design of such algorithms, as they help defend against the conventional pipelined hardware attack by adding uncertainty to the memory access pattern and execution path. However, there are still structural weaknesses remaining. The calculation of $D S$ in Phase III only depends on the salt string and the $D B[0]$. In the proposed accelerator, all of the 256 possible values of $D S$ are pre-computed and reused by all passwords to remove the uncertainty in the calculation of $D S$. This kind of structural weakness can be avoided if dependency on the password string is involved. For example, future algorithm designers can first add the password string to the message $S$ before repeatedly adding the salt string.

Noticing that much of configuration remains the same for different bitstreams, it is possible to optimize the system by utilizing the partial reconfiguration technology. With partial reconfiguration, the overhead of reconfiguration could be reduced to less than 50 ms. However, it requires a great deal of work or even a new method to make our design compatible with partial reconfiguration, which is hoped to be completed in the future. 


\section{References}

[AKSE18] A Abdelrahman, H Khaled, Eman Shaaban, and Wail S Elkilani. WPA-WPA2 psk cracking implementation on parallel platforms. In 2018 13th International Conference on Computer Engineering and Systems (ICCES), pages 448-453. IEEE, 2018.

[AVWS14] Ayman Abbas, Rian Voß, Lars Wienbrandt, and Manfred Schimmler. An efficient implementation of PBKDF2 with RIPEMD-160 on multiple FPGAs. In 2014 20th IEEE International Conference on Parallel and Distributed Systems (ICPADS), pages 454-461. IEEE, 2014.

[CEES14] Louise H Crockett, Ross A Elliot, Martin A Enderwitz, and Robert W Stewart. The Zynq Book: Embedded Processing with the ARM Cortex-A9 on the Xilinx Zynq-7000 All Programmable SoC. Strathclyde Academic Media, 2014.

[CKSV08] Ricardo Chaves, Georgi Kuzmanov, Leonel Sousa, and Stamatis Vassiliadis. Cost-efficient SHA hardware accelerators. IEEE Transactions on Very Large Scale Integration (VLSI) Systems, 16(8):999-1008, 2008.

[Dan15] Quynh H Dang. Secure hash standard. Technical report, 2015.

[DGK $\left.{ }^{+} 12\right]$ Markus Dürmuth, Tim Güneysu, Markus Kasper, Christof Paar, Tolga Yalcin, and Ralf Zimmermann. Evaluation of standardized password-based key derivation against parallel processing platforms. In European Symposium on Research in Computer Security, pages 716-733. Springer, 2012.

[DK14] Markus Dürmuth and Thorsten Kranz. On password guessing with GPUs and FPGAs. In International Conference on Passwords, pages 19-38. Springer, 2014 .

[has20] Hashcat: Advanced password recovery. https://hashcat.net/hashcat/, 2020. [Online; Accessed 9-July-2020].

[HK17] Ignatius Leo Sri Hendarto and Yusuf Kurniawan. Performance factors of a CUDA GPU parallel program: A case study on a PDF password cracking brute-force algorithm. In 2017 International Conference on Computer, Control, Informatics and its Applications (IC3INA), pages 35-40. IEEE, 2017.

[Int20] Intel. Intel Stratix 10 SX SoC FPGAs. https://www.intel.com/content/ www/us/en/products/programmable/soc/stratix-10.html, 2020. [Online; Accessed 9-July-2020].

[Jos06] Simon Josefsson. The base16, base32, and base64 data encodings. 2006.

$\left[\mathrm{KMB}^{+} 16\right]$ Markus Kammerstetter, Markus Muellner, Daniel Burian, Christian Kudera, and Wolfgang Kastner. Efficient high-speed WPA2 brute force attacks using scalable low-cost FPGA clustering [extended version]. arXiv preprint arXiv:1605.07819, 2016.

[Lam81] Leslie Lamport. Password authentication with insecure communication. Communications of the ACM, 24(11):770-772, 1981.

$\left[\mathrm{LCL}^{+} 16\right]$ Xiaochao Li, Chunhui Cao, Pengtao Li, Shuli Shen, Yihui Chen, and Lin Li. Energy-efficient hardware implementation of LUKS PBKDF2 with AES on FPGA. In 2016 IEEE Trustcom/BigDataSE/ISPA, pages 402-409. IEEE, 2016. 
[LLD19] Peng Liu, Shunbin Li, and Qingyuan Ding. An energy-efficient accelerator based on hybrid CPU-FPGA devices for password recovery. IEEE Transactions on Computers, 68(2):170-181, 2019.

[Mag18] Magnumripper. John the ripper. https://github.com/magnumripper/ JohnTheRipper/tree/bleeding-jumbo/src/ztex/fpga-sha256crypt, 2018. [Online; Accessed 9-July-2020].

[Mar08] Simon Marechal. Advances in password cracking. Journal in Computer Virology, 4(1):73-81, 2008.

[MCMM06] Robert P McEvoy, Francis M Crowe, Colin C Murphy, and William P Marnane. Optimisation of the SHA-2 family of hash functions on FPGAs. In IEEE Computer Society Annual Symposium on Emerging VLSI Technologies and Architectures (ISVLSI), pages 317-322. IEEE, 2006.

[MK14] Katja Malvoni and Josip Knezovic. Are your passwords safe: Energy-efficient bcrypt cracking with low-cost parallel hardware. In 8th USENIX Workshop on Offensive Technologies (WOOT), 2014.

[Oec03] Philippe Oechslin. Making a faster cryptanalytic time-memory trade-off. In Annual International Cryptology Conference, pages 617-630. Springer, 2003.

$\left[\mathrm{QGG}^{+}{ }^{16}\right.$ Weidong Qiu, Zheng Gong, Yidong Guo, Bozhong Liu, Xiaoming Tang, and Yuheng Yuan. GPU-based high performance password recovery technique for hash functions. Journal of Information Science and Engineering, 32(1):97-112, 2016.

[RVS15] Manoj D Rote, N Vijendran, and David Selvakumar. High performance SHA-2 core using the round pipelined technique. In IEEE International Conference on Electronics, Computing and Communication Technologies (CONECCT), pages 1-6. IEEE, 2015.

[Xil16] Xilinx. Zynq-7000 all programmable SoC. https://www.xilinx.com/ support/documentation/product-briefs/zynq-7000-product-brief . pdf, 2016. [Online; Accessed 9-July-2020]. 


\section{Appendix A The scalable Zynq-based cluster}

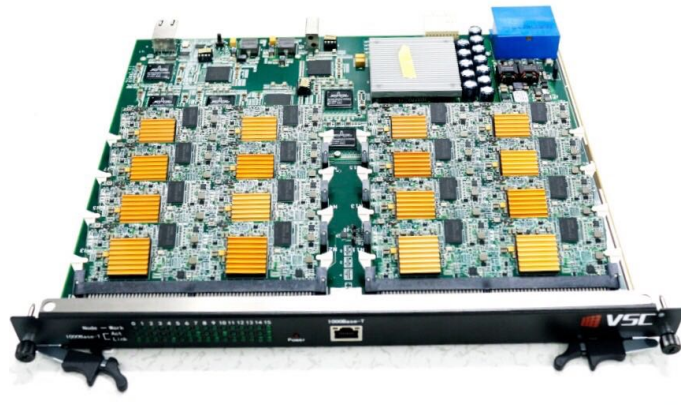

Figure A1: Accelerating cluster with 16 Zynq XC7Z030-3 SoCs.

\section{Appendix B The architecture of the sha256crypt acceler- ator in $\mathrm{JtR}$}

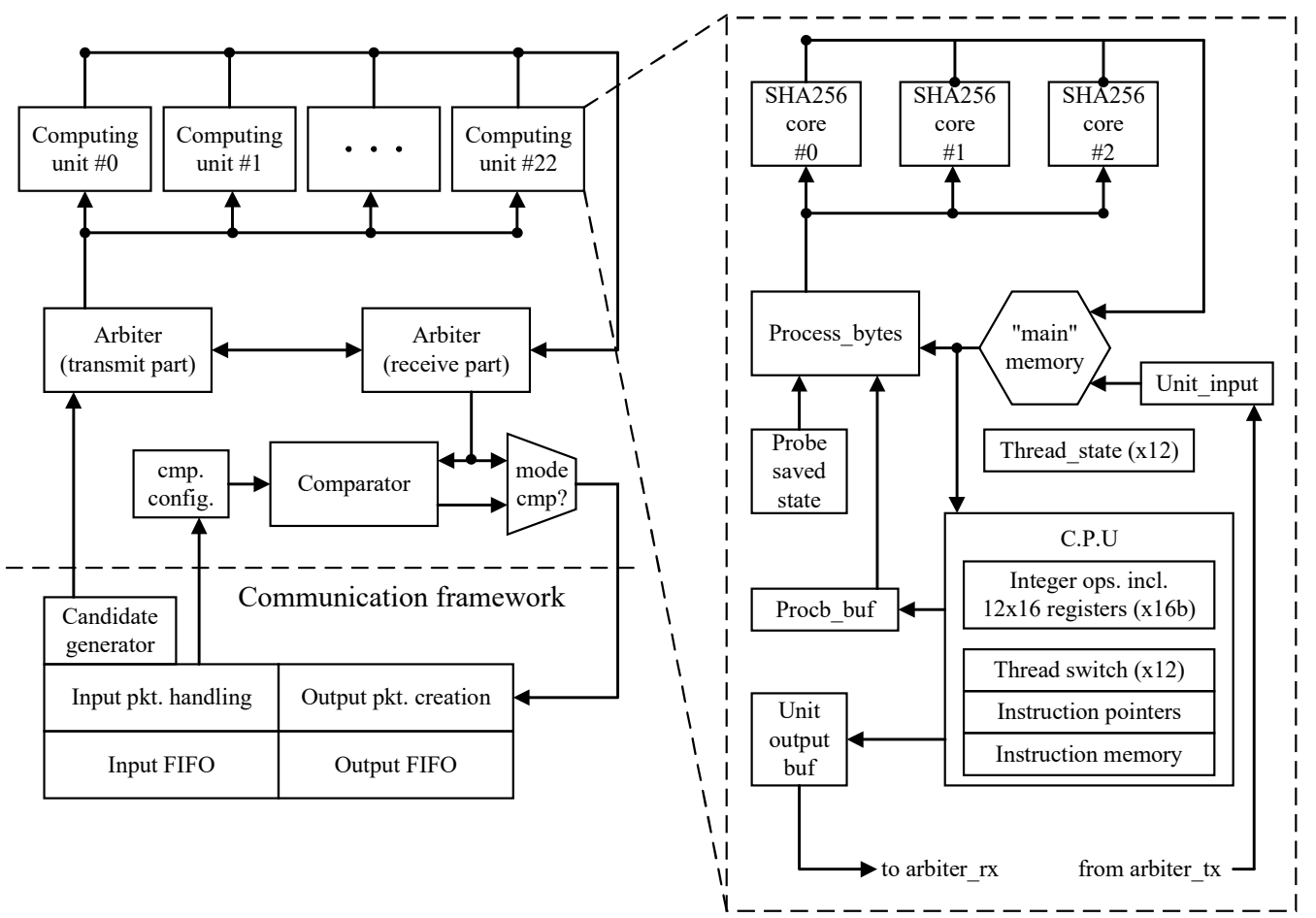

Figure B1: The detailed architecture of the sha256crypt accelerator in JtR. 